\title{
METRIC DIMENSION AND UNCERTAINTY OF TRAVERSING ROBOTS IN A NETWORK
}

\author{
Manjusha $\mathrm{R}^{1}$ andSunny Kuriakose $\mathrm{A}^{2}$ \\ ${ }^{1}$ Lecturer, Amrita Vishwavidyapeetam, Amritapuri, Kollam, Kerala, India - 690525 \\ ${ }^{2}$ Dean, Federal Institute of Science and Technology, Angamaly, Kerala, India -683577
}

\begin{abstract}
Metric dimension in graph theory has many applications in the real world. It has been applied to the optimization problems in complex networks, analyzing electrical networks; show the business relations, robotics, control of production processes etc. This paper studies the metric dimension of graphs with respect to contraction and its bijection between them. Also an algorithm to avoid the overlapping between the robots in a network is introduced.
\end{abstract}

\section{KEYWORDS}

Metric dimension, Contraction, Bijection, Overlapping, Cardinal number.

\section{INTRODUCTION}

If $\mathrm{G}$ is a connected graph with vertex set $V(G)=\left\{v_{1}, v_{2}, \ldots, v_{i}, \ldots, v_{m}\right\}, i=1,2, \ldots, m$ where $m \geq n$ and let $W=\left\{v_{1}, v_{2}, \ldots, v_{j}, \ldots, v_{n}\right\}, j=1,2, \ldots, n$ be an ordered set of vertices of $\mathrm{G}$ and $v$ be a vertex of $\mathrm{G}$. The coordinate of $v$ with respect to $W$ is the k-tuple $\left(d\left(v, v_{1}\right), d\left(v, v_{2}\right), \ldots, d\left(v, v_{n}\right)\right)$. If distinct vertices of $\mathrm{G}$ have distinct co-ordinateswith respect to $W$, then $W$ is called a resolving set or location set for $\mathrm{G}$. A resolving set of minimum cardinality is called a basis for $\mathrm{G}$ and this cardinality is called the metric dimension or location number of $\mathrm{G}$ and is denoted by $\operatorname{dim}(\mathrm{G})$ or $\beta(G)$

The concepts of graph theory have been used to describe navigation in network. In a network, each place represented as nodes in a graph, and edges denote the connections between places. The places or nodes of a network where we place the machines (robots) are called landmarks. The minimum number of machines required to locate each and node of the network is termed as metric dimension and the set of all minimum possible number of landmarks constitute metric basis.

The concept of metric dimension was introduced by P. J.Slater in [2] and studied independently by Harary and Melter in [3]. Applications of navigation of robots in networks were discussed in [4]. Applications to problems of pattern recognition and image processing, which involving the use of hierarchical structures were done in [5]. Besides,Kuller et.al.[6]provideda formula and a linear time algorithm for computing the metric dimension of a tree in [1]. On the other hand, Chartrand et.al.in [7] characterized the graph with metric dimension $1, \mathrm{n}-1$ and $\mathrm{n}-2$ and the tight bound on the metric dimension of unicyclic graphs[8]. Shanmukha and Sooryanarayana $[9,10]$ computed the parameters for wheels, graphs constructed by joining wheels with paths, complete graphs etc. In 1960's Paul Erdos defined the dimension of a graph and stated some related problems and unsolved problems in [11]. The metric dimension of the Cartesian products of graph has been studied by Peters-Fransen and Oellermann [13]. 
International Journal on Applications of Graph Theory in Wireless Ad hoc Networks and Sensor Networks(GRAPH-HOC) Vol.7, No.2/3, September 2015

The metric dimension of various classes of graphs was computed in $[3,4,5,9,10]$.

\section{PRELIMINARIES}

This section summarizes basic definitions and results required in subsequent sections.

\subsection{Definition}

A graph $G=(V, E)$ is an ordered pair consisting of a nonempty set of vertices, $V=V(G)$ and a set of edges, $E=E(G)$. If the endpoints of an edge are equal then it is called a loop and edges having the same pair of endpoints arte called parallel edges. A graph having no loops and parallel edges is a simple graph. A subgraph of a graph $G$ is a graph $H$ such that $V(H) \subseteq V(G)$ and $E(H) \subseteq E(G)$.

Two vertices are said to be adjacent if there is an edgejoining them. The number of vertices in $V(G)$ adjacent to $v$ is the degree of $v$, denoted by $\operatorname{deg}(v)$.

A path is a sequence of distinct vertices $u=v_{o}, v_{1}, \ldots, v_{n}=v$ so that $v_{i-1}$ is adjacent to $v_{i}$ for all $i, 1 \leq i \leq n$, such a path is said to be of length $n$ and if $u=v$ then it becomes a cycle of length $n$ denoted by $C_{n}$.

A connected graph is the one in which there is a path between every two vertices. If each pair of vertices is adjacent then it is called complete graph.

A connected acyclic graph is called tree. A spanning subgraph of $G$ is a subgraph with vertex set $V(G)$ and spanning tree is a spanning subgraph that is a tree.

Let $e=(u, v)$ be an edge in $G$. The contraction of edge $e$ is the replacement of $u$ and $v$ with a single vertex and the edges other than $e$ incident with this single vertex are those edges that were incident with $u$ or $v$. The resulting graph is denoted by G. $e$ and $|E(G . e)|=|E(G)|-1$.

\subsection{Definition}

Coordinate of a vertex $v_{i}$ is represented by an n-tuple $\left(m_{i 1}, m_{i 2}, \ldots, m_{i n}\right), i=1,2, \ldots, m$. In particular for the vertex $v_{1}$ the coordinate is $\left(m_{11}, m_{12}, \ldots, m_{1 n}\right)$ where $m_{11}=d\left(v_{1}, v_{1}\right), m_{12}=d\left(v_{1}, v_{2}\right)$ and so on.

\subsection{Definition}

Cardinal number of a basis element is denoted by $\mathrm{Ca}\left(v_{j}\right), j=1,2, \ldots, n, v_{j} \in W$ and is defines as the number of vertices of G identified by $v_{j}$ with respect to $d\left(v_{i}, v_{j}\right)=l, i=1,2, \ldots, m$.

For example, consider the graph given in Figure1 that has metric dimension two with respect to the basis $W=\{a, b\}$. 


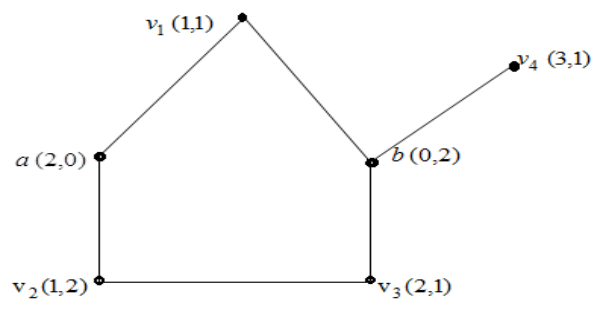

Figure 1

In the figure $C a(a)=2$ and $C a(b)=3$ with respect to $d\left(v_{i}, v_{j}\right)=1$.

\subsection{Definition}

Two vertices $u$ and $v$ in $W$ are said to be overlapping with each other if $C a(u)=C a(v)$ with respect to $d\left(v_{i}, u\right)=d\left(v_{i}, v\right)=l$.

In Figure $1 a$ and $b$ are overlapping with respect to $d\left(v_{1}, a\right)=d\left(v_{1}, b\right)=1$.

\subsection{Robotic Assignment}

Let $W=\left\{v_{1}, v_{2}, \ldots, v_{j}, \ldots, v_{n}\right\}, j=1,2, \ldots, n$ be the basis and $V(G)=\left\{v_{1}, v_{2}, \ldots, v_{i}, \ldots, v_{m}\right\}, i=1,2, \ldots, m$ be the vertex set of $G$. For a vertex $v_{i} \in V(G)$, the coordinate is $\left(m_{i 1}, m_{i 2}, \ldots, m_{i n}\right), i=1,2, \ldots, m$. We can assign the basis element (Robot) $v_{j}$ if $\operatorname{Min}\left(m_{i 1}, m_{i 2}, \ldots, m_{i n}\right)=m_{i j}$ for a particular $j$.

Example 1: In Figure 1 the coordinate of vertex $v_{2}$ is $(1,2)$ with respect to the basis $W=\{a, b\}$. Here, $\operatorname{Min}(1,2)=1$, therefore basis element $a$ is assigned to $v_{2}$.

Note 1: Suppose there is an alternative minima for $j$ and $j+1$. Then we can assign $v_{j}$ to $v_{i}$ if $\mathrm{Ca}\left(v_{j}\right)<\mathrm{Ca}\left(v_{j+1}\right)$.

In Figure 1 the coordinate of $v_{1}$ is $(1,1)$. Therefore $d\left(a, v_{1}\right)=d\left(b, v_{1}\right)=1$ and here we can assign $a$ to $v_{1}$ since $C a(a)<C a(b)$ where $C a(a)=2$ and $C a(b)=3$ with respect to $l=1$.

Note 2: If $\mathrm{Ca}(a)=\mathrm{Ca}(b)$ then arbitrarily any one can assigned to a vertex $v_{i}$ with respect to $d\left(a, v_{i}\right)=d\left(b, v_{i}\right)=1$. In Figure 2 for the vertex $v, \operatorname{Min}(1,1)=1$ and $\operatorname{Ca}(a)=C a(b)=1$ with respect to $l=1$. So assign $a$ or $b$ to $v$.

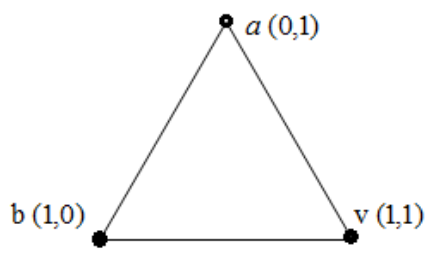

Figure 2 


\subsection{Robotic Assignment Subgraph}

After making the Robotic Assignment we may obtain a spanning subgraph called Robotic Assignment spanning subgraph(RASS). Figure 3 represents the RASS for the graph given in figure 1.

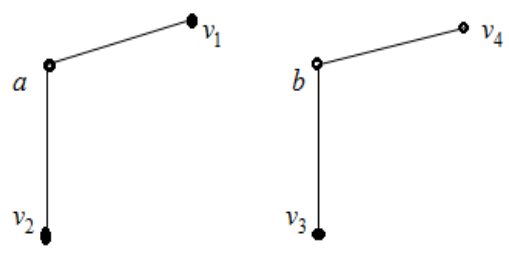

Figure 3

Now we recall a few results already published in $[15,16]$

\subsection{Theorem[7]}

The metric dimension of graph $G$ is 1 if and only if $G$ is a path.

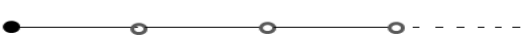

Figure 4

\subsection{Theorem [7]}

The metric dimension of a complete graph with $n$ vertices is $n-1$ where $n>1$.

\subsection{Theorem [14]}

Let $\tau(G)$ denote the number of spanning trees of a graph $G$. If $e \in E(G)$ is not a loop then $\tau(G)=\tau(G-e)+\tau(G . e)$.

\section{MAIN RESULTS}

\subsection{Theorem}

Consider the graph $K_{n}$ with $n$ vertices $v_{1}, v_{2}, \ldots, v_{n}$, then $\beta\left(K_{n} . e\right)=n-2$. where $K_{n} . e$ denote the contracted graph having no loops and parallel edges.

Proof: We have $\beta\left(K_{n}\right)=n-1$. Let $e=u v$ be an edge in $K_{n}$ where $u$ and $v$ are adjacent to every other $n-2$ vertices. Consider the contracted graph $K_{n} . e$. Since every vertex in $K_{n} . e$ are adjacent to the other $n-2$ vertices. Here the edge $e \notin E\left(K_{n} . e\right)$ and the vertices $u$ and $v$ are replaced by a single vertex say $w^{*}$. Evidently the remaining $n-2$ vertices must adjacent to $w^{*}$ 
since both $u$ and $v$ are adjacent to those vertices. Then the simple graph $K_{n}$.e contains exactly $n-1$ vertices and it should be $K_{n-1}$. Clearly $\beta\left(K_{n-1}\right)=n-1-1=n-2$. Hence the proof.

Figure 5 represents $K_{4}$ and its contraction (simple graph) with the edge $e$.

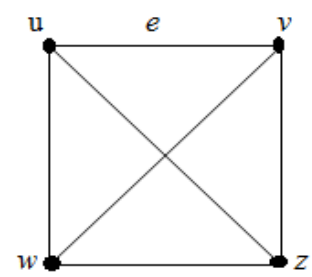

$K_{4}$

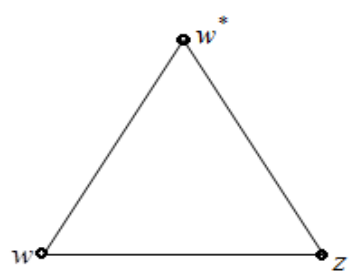

Simple graph of $K_{4} \cdot$ e

Figure 5

For large scale computations, network models consist of several nodes and can place uniquely a minimum number of Robots to identify them. But in the case of optimization we cannot assign two machines (Robots) to the same node. The following algorithm explains that how we can avoid the overlapping between the machines.

\subsection{Theorem (Algorithm)}

Let $G$ be a simple connected graph with $V(G)=\left\{v_{1}, v_{2}, \ldots, v_{i}, \ldots, v_{m}\right\}, i=1,2, \ldots, m$. and $W=\left\{a_{1}, a_{2}, \ldots, a_{j}, \ldots, a_{n}\right\}, j=1,2, \ldots, n$ be the basis of $G$ where $a_{j}=v_{i}$ for some $i$ and $j=1,2, \ldots, n \& n \leq m$ we can find any two adjacent vertices in $G$ since it is connected.

The following steps yields a Robotic assignment subgraph for $G$.

Step 1: If $d\left(a_{1}, v_{i}\right)<d\left(a_{j}, v_{i}\right)$ for $j=2,3, \ldots, n$ then assign $a_{1}$ to $v_{i}$ and vice versa for $i=1,2, \ldots, m$ and $v_{i} \notin W$.

Step 2: Let $a_{1}$ is adjacent to any of the vertex $v_{i}$ for some $i$ that is not in $W$ and $d\left(a_{1}, v_{i}\right)=1$. Consider all other $a_{j}$ such that $d\left(a_{j}, v_{i}\right)=1$.

Step 3: If $C a\left(a_{1}\right)<C a\left(a_{j}\right)$ for $j=2,3, \ldots, n$ then assign $a_{1}$ to $v_{i}$ and add the edge $\left(a_{1}, v_{i}\right)$ or in other words $v_{i}$ is identified by the basis element $a_{1}$.

Step 4: Now take $d\left(a_{j}, v_{i}\right)=2$ for some $j$ and $v_{i}$ is ma vertex which is not previously identified by any of the basis element. Consider all $a_{k}, k=1,2, \ldots, j-1, j+1, \ldots, n$ at which $d\left(a_{k}, v_{i}\right)=2$. If $C a\left(a_{j}\right)<C a\left(a_{k}\right)$ for $k=1,2, \ldots, j-1, j+1, \ldots, n$ then assign $a_{j}$ to $v_{i}$ and join the path from $a_{j}$ to $v_{i}$.

Step 5: Continue the process till all the vertices in $G$ is identified by the any of the basis element in $W$.

Step 6: Joining an edge or path in this way we may obtain a connected or disconnected graph called Robotic assignment subgraph of $G$ obviously it is spanning subgraph of $G$.

It can be easily verified that the graph in Figure 3 represents the Robotic assignment subgraph of the graph in Figure 1. 
Note: If the Robotic assignment subgraph is not connected then adjoin the edge or path between any two vertices which are not in $W$ to make it a connected spanning subgraph. This is possible since the graph is connected. Hence we obtain a spanning tree for $G$. The following figure represents the Robotic spanning tree $S$ of the graph in Figure 1.

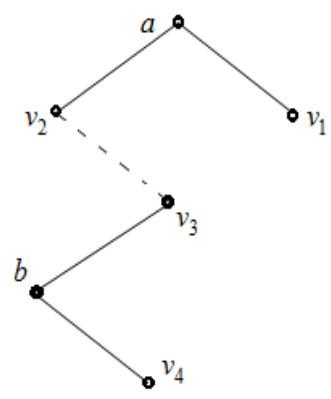

Figure 6

\subsection{Adjacency matrix of $S$}

It is interesting to know about the paths between basis elements and vertices of graphs. We know that the each entry in adjacency matrix $[S]_{n \times n}$ gives the path of length one between any two vertices in the graph, entry in $[S]^{2}$ gives the path of length two between any two matrices and so on. We are concentrating on the different path from basis elements to the vertices in the graph that are not in the basis. If we consider a sub matrix $[S]_{n \times n}$ of order $m \times n, m \leq n$ then each entry in that matrix gives path from Robots to nodes in the network. One can easily show that the diagonal of the matrices $[S]^{2}$ and $M M^{T}$ are equal where $M$ is the incidence matrix of $S$.

\subsection{Example}

Suppose the following graph represents the Robotic spanning tree $S$ of some graph $G$ and its adjacency matrix and incidence matrix are given in Figure 7.

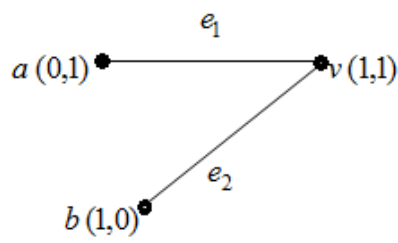

Figure 7 
It is shown that the diagonals of $[S]^{2}$ and $M M^{T}$ are same. That is we can easily find the route of length two from a robot to itself.

$$
\begin{aligned}
& {[S]=\left[\begin{array}{lll}
0 & 0 & 1 \\
0 & 0 & 1 \\
1 & 1 & 0
\end{array}\right] M=\left[\begin{array}{ll}
1 & 0 \\
0 & 1 \\
1 & 1
\end{array}\right]} \\
& {[S]^{2}=\left[\begin{array}{lll}
0 & 0 & 1 \\
0 & 0 & 1 \\
1 & 1 & 0
\end{array}\right]\left[\begin{array}{lll}
0 & 0 & 1 \\
0 & 0 & 1 \\
1 & 1 & 0
\end{array}\right]=\left[\begin{array}{lll}
1 & 1 & 0 \\
0 & 1 & 0 \\
0 & 0 & 2
\end{array}\right]} \\
& M M^{T}=\left[\begin{array}{ll}
1 & 0 \\
0 & 1 \\
1 & 1
\end{array}\right]\left[\begin{array}{lll}
1 & 0 & 1 \\
0 & 1 & 1
\end{array}\right]=\left[\begin{array}{lll}
1 & 0 & 1 \\
0 & 1 & 1 \\
1 & 1 & 2
\end{array}\right]
\end{aligned}
$$

Since $S$ is a spanning tree the number of different spanning trees of $S, \tau(S)=1$. The following theorem shows that there is a one to one correspondence between $E(S)$ and $\tau(S$.e) with respect to a contraction. By means of contraction actually we reduce the cardinal number of each basis element. So contraction has great importance in the routing of complex networks.

\subsection{Theorem}

If $S$ is the Robotic spanning tree of a graph $G$ and S.e is the contraction with respect to nonloop $e$ then there exists a bijection between the edge set of $S$ and $\tau(S . e)$.

Proof: Assume that no two basis elements in $S$ are adjacent. For each edge $e \in E(S)$, the contraction $S$.e must be a tree since $S$ itself a tree. Now $S$. e contains E(S)-1 edges and V(S)-1 vertices. If $S$ contains medges then corresponding to medges we get $\mathrm{m}$ trees with respect to a contraction $S$.e. Since each tree of $S$. e arises for exactly on edge from $S$ and $S$.e itself a tree means $\tau(S$. e $)=1$. That means each contracted edge gives one and only one spanning tree of $S$.e . Thus there is a bijection between $\mathrm{E}(\mathrm{S})$ and $\tau(S$.e) .

\subsection{Example}

Consider the Robotic spanning tree in the Figure 6. The figure given below shows the correspondence stated in the above theorem. 
International Journal on Applications of Graph Theory in Wireless Ad hoc Networks and Sensor Networks(GRAPH-HOC) Vol.7, No.2/3, September 2015
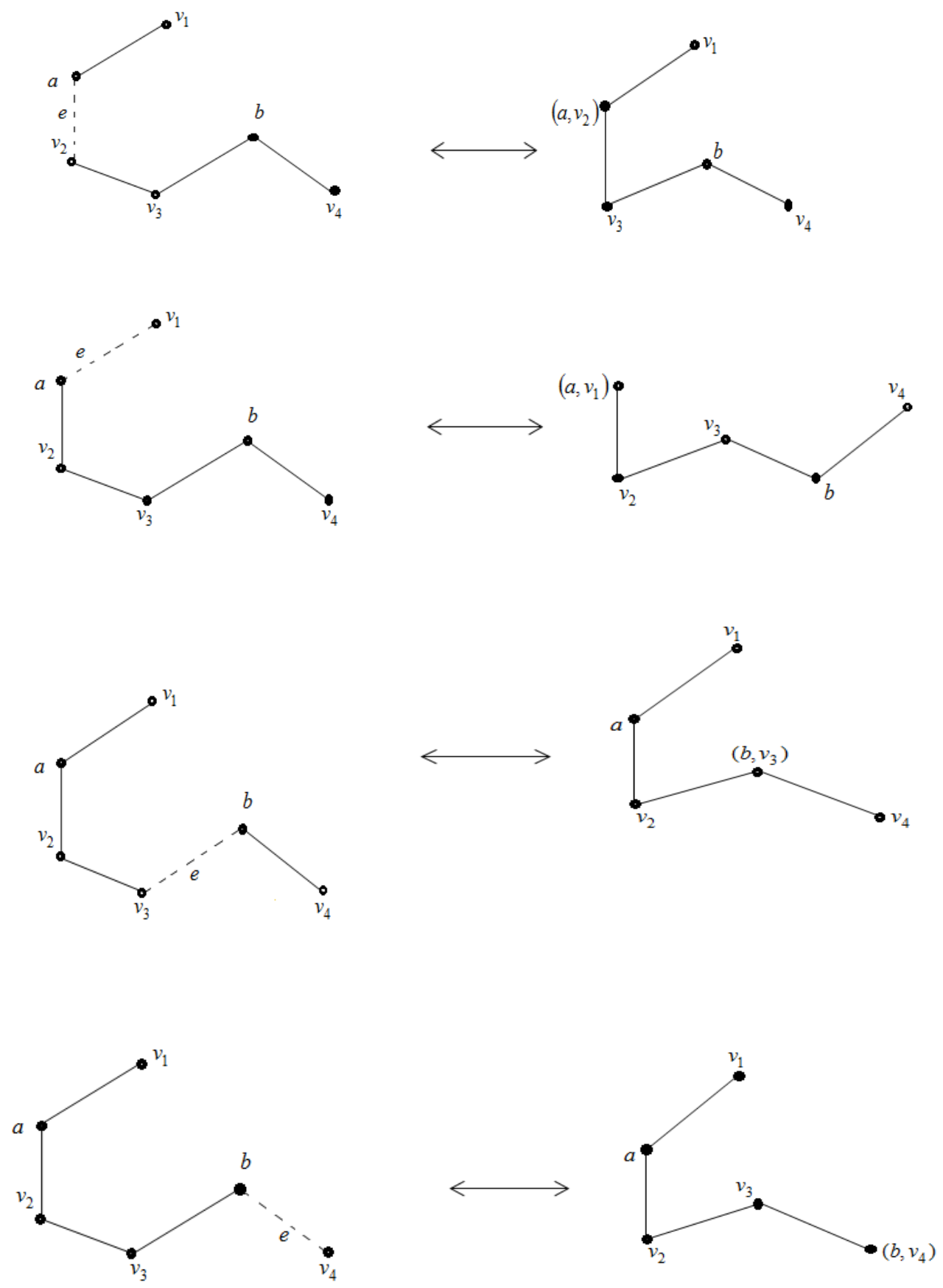

Figure 8 


\section{CONCLUSION}

In this paper we studied the metric dimension of graphs with respect to contraction and its bijection between them. The algorithm proposed is used to avoid the overlapping between the robots in a network. This is applied in Robotic Assignment spanning subgraph in a complex network and provides an optimization through an algorithm in which the routing of basis elements helps in solving some complicated networks.

\section{REFERENCES}

[1] J. Caceres, C. Hernado, M. Mora, I. M. Pelayo, M. L. Puertas, C. Seara and D. R. Wood, "On the Metric Dimension of Some Families of Graphs," Electronic Notes in DiscreteMathematics, Vol. 22, 2005, pp. 129-133.

[2] P. J. Slater. Leaves of trees. In: Proc. 6th Southeastern Conf. on Combinatorics, Graph Theory and Computing, 14, pp 549-559, 1975.

[3] F. Harary and R. A. Melter, "On the Metric Dimension of a Graph," ArsCombinatorica, Vol. 2, 1976, pp. 191-195.

[4] I. Javaid, M. T. Rahim and K. Ali, "Families of Regular Graphs with constant Metric Dimension," UtilitasMathematica, Vol. 75, 2008, pp. 21-33.

[5] S. Khuller, B. Raghavachari and A. Rosenfeld, "Localization in Graphs," Technical Report CS-Tr3326, University of Maryland at College Park, 1994.

[6] S. Khuller, B. Raghavachari and A. Rosenfeld. Landmarks in Graphs. Discrete Appl.Math, 70(3), pp. 217-229, 1996.

[7] G. Chartrand, L. Eroh, M. A. Johnson, and O. R. Oellermann. Resolvability in graphs andMetric Dimension of Graph. Discrete Appl. Math., 105(1-3), pp. 99-113, 2000.

[8] C. Poisson and P. Zhang. The metric dimension of unicyclic graphs. J. Combin. Math.Combin. Compute. 40, pp. 17-32, 2002.

[9] B Sooryanaranyana, B. Shanmuka, A Note on metric dimension, Far. East Journal of Applied Mathematics, 5, 331-339, 2001.

[10] B Sooryanaranyana, B. Shanmuka, Metric dimension of a wheel, Far. East Journal of Applied Mathematics, 6, 8(3), 217-229, 2002.

[11] P. Erdos, "On sets of distances of ' $n$ ' points in Euclidean space," Publ. Math. Inst. Hung.Acad. Sci, 5 (1960), 165-169.

[12] A. Sebo, E. Tannier, "On metric generators of Graphs, Mathematics of Operation Research , 29(2) (2004) 383-393.

[13] J. Peters-Fransen and O. R. Oellermann. The metric dimension of Cartesian products of Graphs. Util. Math., 69, pp 33-41, 2006.

[14] Douglas B. West, "Introduction to Graph Theory, Second Edition, University of Illions-Urbana.

[15] Manjusha. R, Dr. Sunny Kuriakose A 'On metric dimension of some special graphs and its isomorphism', University Grant Commission of India sponsored National conference on Fuzzy Logic and its Applications to Computer Science,2014.

[16] Manjusha. R, Dr. Sunny Kuriakose A “An Application of Gd-Metric Spaces and Metric Dimension of Graphs" International Journal on Applications of Graph Theory in Wireless Ad hoc Networks and Sensor Networks(GRAPH-HOC) Vol.7, No.1, March 2015. 